\title{
Modeling Object Pursuit for 3D Interactive Tasks in Virtual Reality
}

\author{
Lei Liu* $\quad$ Robert van Liere ${ }^{\dagger}$
}

CWI, Amsterdam, NL

\begin{abstract}
Models of interaction tasks are quantitative descriptions of relationships between human temporal performance and the spatial characteristics of the interactive tasks. Examples include Fitts' law for modeling the pointing task and Accot and Zhai's steering law for the path steering task, etc. Models can be used as guidelines to design efficient user interfaces and quantitatively evaluate interaction techniques and input devices.

In this paper, we introduce a 3D object pursuit interaction task, in which users are required to continuously track a moving target in a virtual environment. The entire movement of the task is broken into a tracking phase and a correction phase. For each phase, we propose a model that has been verified by two experiments. As the experimental results show, the time for the tracking phase is fixed once a task has been established, while the time for the correction phase usually varies according to some characteristics of the task. It can be modeled as a function of path length, target width and the velocity with which the target moves.

The proposed model can be used to quantitatively evaluate the efficiency of user interfaces that involve the interaction with moving objects.
\end{abstract}

Index Terms: H5.1 [Information Interfaces and Presentation]: Multimedia Information Systems-Artificial, augmented, and virtual realities; H5.2 [Information Interfaces and Presentation]: User Interfaces-Ergonomics, Theory and methods

\section{INTRODUCTION}

In physiology, pursuit is defined as the action of the eye in following a moving object. In this paper, we introduce the term to describe an interaction task, in which users are required to continuously track a 3D moving object with a tracker held in their hands in a virtual environment. Object pursuit tasks can be found extensively in gaming, video surveillance system, air traffic control system, etc. A shooting game with moving targets, for example, is a typical pursuit task. As with pointing and path steering, object pursuit can be considered to be a primitive but unique interaction task. One major characteristic it differs from pointing and steering tasks is that in an object pursuit task, subjects interact with a non-stationary target which is usually kept stationary in a pointing or a path steering task.

In human computer interaction, modeling is the approach to quantify interaction tasks. Widely used and well known examples include Fitts' law [7] for modeling pointing task and Accot and Zhai's steering law [1] for path steering task, etc. Models formally describe quantitative relationships between human temporal performance and the spatial characteristics of an interactive task. Interaction models have guided user interface designers in designing efficient user interfaces $[20,16]$. They also provide a quantitative approach to evaluate the efficiency and performance between e.g. input devices [17, 2] or interaction techniques [15], etc.

\footnotetext{
e-mail: Lei.Liu@cwi.nl

†e-mail: Robert.van.Liere@cwi.nl
}

The goal of this paper is to derive a model for the 3D objec$\mathrm{t}$ pursuit interactive task. The complete object pursuit movement is divided into a tracking phase and a correction phase. For each phase, we propose a model to describe the temporal information. The time for the tracking phase is fixed once a task has been established, while the time for the correction phase usually varies with the characteristics of the task. The correction time can be modeled as a function of the size and velocity of the target and the path length crossed by the target. The model has been verified by two experiments with repeated measures design.

As indicated by the experimental results, the growth of the velocity with which the target moves usually results in longer correction time, but shorter tracking time. Therefore, the selection of the target velocity is important on reducing the total time (tracking time + correction time) of an object pursuit task. By analyzing the proposed model, we manage to derive a velocity, with which the total movement time reaches its minimum. This velocity, described as a function of path length and target width, can be used as a guide in designing user interfaces with moving targets.

Furthermore, there are several aspects that we can learn from modeling the object pursuit task for a general interaction task. The way that the influence of path length and target width combines in object pursuit tasks very much resembles the way how it affects pointing and path steering tasks. This fact may be interpreted as evidence of such combination in modeling a general interactive task. In addition, the model also reveals that at least one time-related factor, besides spatial factors, is required to model an interactive task that involves a non-stationary target. The time-related factor, e.g. the target velocity in the object pursuit task, plays such an important part that it may even become an overwhelming factor in affecting users' performance time. We suggest that both time and space-related factors should be taken into account when modeling an interaction task with moving targets.

The main contributions of this work focus on the following points:

- We propose to analyze an object pursuit task in such a way that the complete movement should be broken into a tracking phase and a correction phase (see Section 3.3 for details);

- We point out that the time for the tracking phase is fixed if the task has been established. It can be described by

$$
T_{\text {tracking }}=\frac{L}{v}
$$

when the target moves a distance $L$ with a uniform velocity $v$ (see Section 4);

- We propose a model for the time of the correction phase, which has been experimentally verified. It has the following form (see Section 4.2.3):

$$
\log \left(T_{\text {correction }}\right)=a+b \frac{L}{W}+c v
$$

where $a, b$ and $c$ are the empirically determined constants, and $L, W$ and $v$ are the path length, target width and target velocity. 
- We manage to derive an optimal target velocity with which the total time ( $\left.T_{\text {tracking }}+T_{\text {correction }}\right)$ can be minimized. This velocity can be expressed with path length and target width (see Section 5).

\section{Related Work}

\subsection{Pursuit Task}

Pursuit is one of the important human skills that have been used, for example, to differentiate normal subjects from psychiatric patients $[12,8,9]$ or to qualify a pilot $[11,19]$. Given the parts of the human body that are used, pursuit can be categorized into eye movement [13, 3], locomotion [4], manual tasks [21], etc. It has been extensively studied in the discipline of psychology, but has never been systematically investigated as an interaction task in human computer interaction. In this paper, we use the term to describe an interaction task that requires subjects to manually steer an input stylus in pursuit of a 3D target that moves with uniform velocity in a virtual environment.

\subsection{Modeling Interaction Task}

In human-computer interaction, there are quite a few wellformalized and accepted quantitative models that can be used as tools to compare the efficiency between interaction techniques or input devices. One of the best known model Fitts' law, proposed by Paul Fitts in 1954 [7], has a long history and a widely applied scope $[22,6,17]$. It predicts that the time required to rapidly move to a target is a function of the distance to and the size of the target. Fitts' law has been used to model the act of pointing in both the physical world [5] and the virtual environment [10, 24]. One common formulation of the law is as follows:

$$
T=a+b \mathrm{ID}=a+b \log _{2}\left(\frac{L}{W}+1\right)
$$

where $a$ and $b$ are experimentally determined constants, $L$ is the distance to the target, and $W$ is the target width. The term $\log _{2}(L / W+1)$ is referred to as the index of difficulty (ID) of a pointing task.

It was not until a few decades later that Accot and Zhai derived the steering law $[1,25]$ from Fitts' law for path steering tasks. The idea of the steering law assumes that a path steering task is composed of an infinitive number of goal crossing tasks, each of which could be separately modeled by Fitts' law. If the path width varies with the path, the generic steering law can be expressed in the following formula:

$$
T_{C}=a+b \mathrm{ID}=a+b \int_{C} \frac{d s}{W(s)}
$$

where $a$ and $b$ are empirically determined constants, $C$ is a curved path, $s$ is the elementary path length along $C$ and $W(s)$ is the path width at path length $s$. If the path width keeps fixed, the steering law can be rewritten as:

$$
T_{C}=a+b \frac{L}{W}
$$

with $L$ and $W$ representing the length and width of the path, respectively.

However, as Liu et al. [14] questioned, it is counterintuitive to describe the steering time only as a function of path length and width. They proposed to involve other factors to the steering law as shown below:

$$
\log (T)=a+b \mathrm{ID}=a+b\left(\log \frac{L}{W}+c \rho\right)
$$

where $I D$ is redefined as $\log (L / W)+c \rho$, introducing the influence of curvature $\rho$ of the steering path, together with length $L$ and width $W$.
It is important to note that the models mentioned above all describe the temporal information with the spatial characteristics of the tasks. In this paper, we use a similar way to propose a model for 3D object pursuit task.

\section{EXPERIMENT}

\subsection{Apparatus and Environment}

The experiment was performed in a desktop virtual environment as shown in Figure 1. The specific apparatus includes:

- a desktop PC equipped with high end GPU,

- a Samsung 67-inch 3D-capable LED DLP HDTV,

- a pair of Crystal Eyes stereoscopic LCD glasses,

- a Polhemus FASTRAK connected with one 6DOF stylus tracker (sampling @ 120Hz),

- a 6DOF ultrasound Logitech head tracker (working @ 60Hz).

During the experiment, the resolution of the Display was set to 1920 $\times 1080 @ 120 \mathrm{~Hz}$. The end-to-end latency was measured to be approximately $80 \mathrm{~ms}$ with the method proposed by Steed [23].

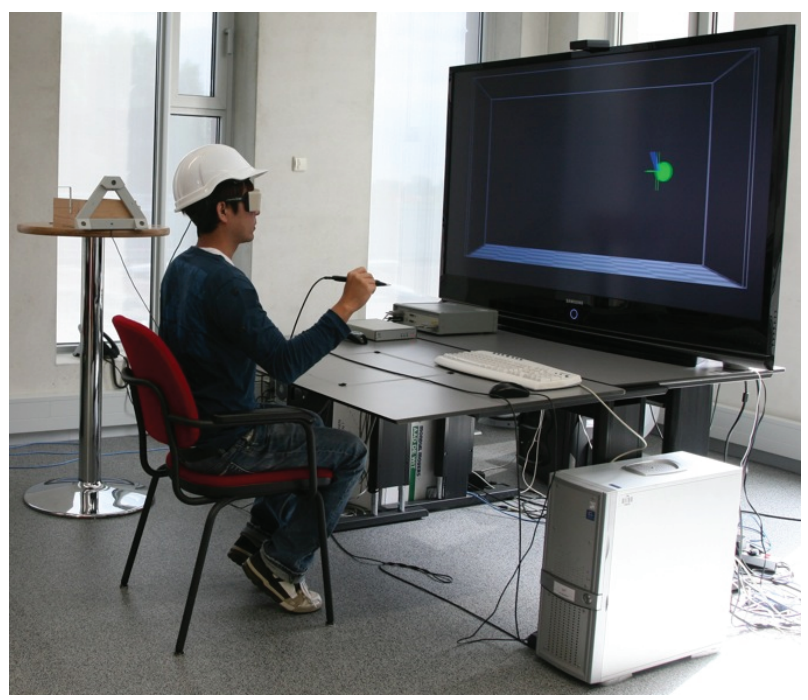

Figure 1: The experimental environment: a head tracked stereo display and a 6DOF input stylus. Several depth cues were created during the experiment, including the stereoscopic viewing, head tracking, head lighting, wire-frame box and the chessboard pattern floor.

The experiment was set up in a non-colocated environment with a distance of $0.65 \mathrm{~m}$ between the visual and motor space (see Figure 2). The control and display ratio was always kept 1 . The origin of the visual space was set to $0.4 \mathrm{~m}$ in front of the display and $0.6 \mathrm{~m}$ above the desktop, while that of the motor space was $0.3 \mathrm{~m}$ ahead of the subject and $0.3 \mathrm{~m}$ above the desktop. Subjects were seated $1.35 \mathrm{~m}$ away from the display and were required not to rest their arms on the table.

\subsection{Subject}

Twenty right-handed subjects voluntarily participated in the experiment. There were six females and fourteen males, aged 24 to 35 . Five of them were invited to perform a pilot study, while the remaining fifteen subjects were instructed to take part in the following main experiment. The experienced and non-experienced subjects were kept in a ratio of 2:3 in both pilot study and main experiment. 


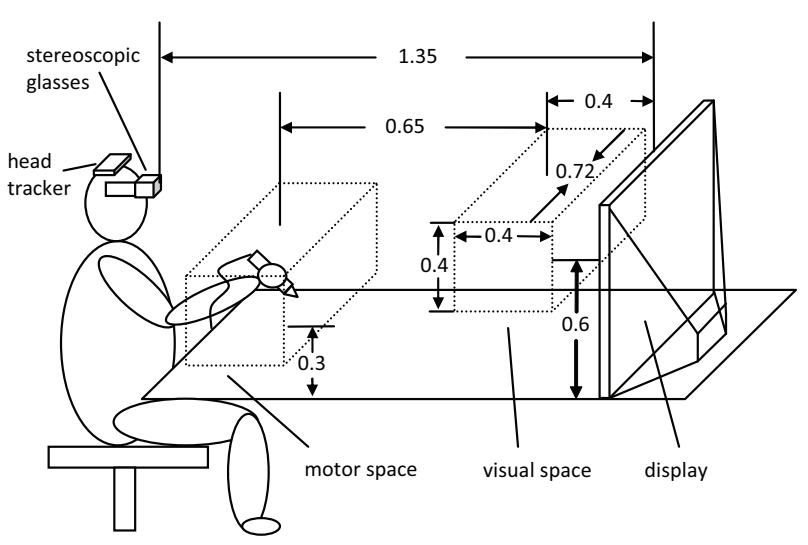

Figure 2: The experimental setup [14] (units: meter): Motor and visual space were not co-located and C-D ratio $=1$.

\subsection{Task}

The experiment was designed to examine the object pursuit tasks performed by the subjects. For this, subjects were required to hold an input stylus, represented as a $3 \mathrm{D}$ pen in the virtual environment, with their dominant hands to trace a target (3D ball) moving in the visual space. To enhance the visual feedback, 3D coordinate axes were attached to the tip of the pen. The space in which the $3 \mathrm{D}$ ball moved were encapsulated in a $0.72 \times 0.4 \times 0.4 \mathrm{~m}^{3}$ (length $\times$ height $\times$ depth) sized wire-frame box, whose floor was covered with a chessboard pattern (See Figure 1 and 2).

In the initial phase, subjects were shown a stationary target ball and both the ball and the axes attached to the pen were colored red. The target ball started to move with a uniform velocity, once the tip of the pen, i.e. the origin of the 3D axes was inside the target ball (see Figure 3, right). Meanwhile, the ball and the axes turned to green. Subjects were asked to track the moving target by keeping the tip of the pen within the ball as possible as they could. If they failed, the moving target stopped, making the axes and the ball red again (see Figure 3, left). Subjects had to correct this movement by steering the pen back to the ball and resuming the tracking where they left off. A trial started when the target ball left the initial position and proceeded until the target ball moved to a predefined destination that was not known to the subjects in advance. Consequently, subjects were shown the number of trials left on the screen.

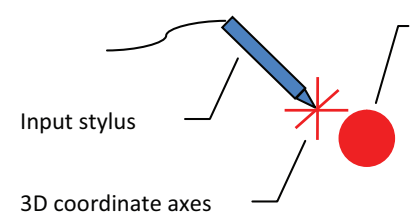

Correction



Tracking
Figure 3: The correction phase (left) and the tracking phase (right) for an object pursuit task.

The time when the tip of the pen was within the target ball and moved with it was defined as the tracking time, i.e. the total time when target ball remained green, while the time when the tip of the pen deviated from target ball and the subject made a correction was defined as the correction time, i.e. the total time when the target ball remained red. The time for completing an object pursuit task, i.e. the sum of the tracking time and the correction time, was defined as the total time.

\subsection{Procedure}

A pilot study was carried out before the main experiment with the purpose of acquiring a proper model and parameter setting for the upcoming main experiment. The design of the pilot study was different from that of the main experiment in a way that it distinguished the effect of velocity of the moving target from that of target width and path length, examining the effect of only a minimum number of independent variables at a time. Therefore, the study was split into four independent pilot studies. In pilot study 1 and 2 where velocity was fixed, we examined linear and circular paths respectively with 2 target widths and 3 path lengths, while in pilot study 3 and 4 where path length and target width were kept constant, linear and circular paths were studied with 10 velocities. As a result, two sizes for the target ball, three lengths for path and five constant velocities of the target ball were selected for the main experiment.

We have conducted two main experiments of repeated measures design, in which two types of paths of the moving target were applied. The target ball always made a uniform motion on a straight path (see Figure 3.4, left) in experiment 1, while it moved along a segment of a circular path (see Figure 3.4, right) with a constant velocity in experiment 2 . The radius of the target ball was set to $0.015 \mathrm{~m}$ and $0.02 \mathrm{~m}$, constraining the movement of the pen to $0.03 \mathrm{~m}$ and $0.04 \mathrm{~m}$ in amplitude. We chose $0.24 \mathrm{~m}, 0.30 \mathrm{~m}$ and $0.36 \mathrm{~m}$ as the path length so that completing an object pursuit task only required the extension of the arm, keeping the body relatively still. The velocity of the moving target, including $0.10,0.15$, $0.20,0.25,0.30 \mathrm{~m} / \mathrm{s}$, was tested to be suitable to track for both nonexperienced and experienced subjects. Each combination of the above parameters was repeated three times, resulting in $2 \times 3 \times 5 \times 3$ (target size $\times$ path length $\times$ motion velocity $\times$ repeats) trials in each experiment and a total number of 180 trials for a subject. To compensate the practice effect, trials were presented in a random order that differed from one subject to another. Subjects were allowed to have a break whenever they suffered from fatigue between trials. This was, however, strictly prohibited during a trial.
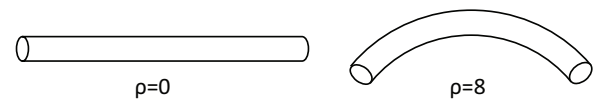

Figure 4: Two types of paths used in Experiment 1 (left) and 2 (right). The curvature of the path, represented by $\rho$, is defined as the reciprocal of the path radius. The paths were not shown to the subjects.

\section{Result}

To model the object pursuit tasks, inspired by Fitts' law and steering law, we chose to statistically explore a relationship between the temporal information, e.g. the tracking time $T_{\text {tracking }}$, correction time $T_{\text {correction }}$ and total time $T_{\text {total }}=T_{\text {tracking }}+T_{\text {correction }}$, and the characteristics of the tasks, e.g. the target size $W$, the velocity of the moving target $v$ and the path length $L$ crossed by the target during a trial.

The tracking time, i.e. the time when the target ball moves with a constant velocity, can be described as:

$$
T_{\text {tracking }}=\frac{L_{1}}{v}+\frac{L_{2}}{v}+\ldots+\frac{L_{n}}{v}=\frac{L}{v}
$$

where $L_{n}$ is the length of the $n$-th path segment crossed by the target ball without a correction. As can be seen, $T_{\text {tracking }}$ is constant if $L$ and $v$ have been fixed.

Different from $T_{\text {tracking }}$, modeling $T_{\text {correction }}$ requires ANOVA and regression analysis. It is, therefore, necessary to verify if the 
data is normally distributed. Figure 4 plots the associated probability density function of $T_{\text {correction }}$ for experiment 1 and 2 , correspondingly. As shown, both histograms considerably deviate from the normal distribution that fits onto the data (right-skewed). A data transformation is needed to bring the density function closer to that of a normal distribution. Our approach was to apply natural logarithmical transformation (base $e$ ) to $T_{\text {correction. }}$. As illustrated in Figure 6, the data after transformation approximately follow a bellshaped density function. The Kolmogorov-Smirnov test shows that the null hypothesis, i.e. the transformed data have a normal distribution, can not be rejected $(h=0)$ at the $5 \%$ significance level in each experiment.
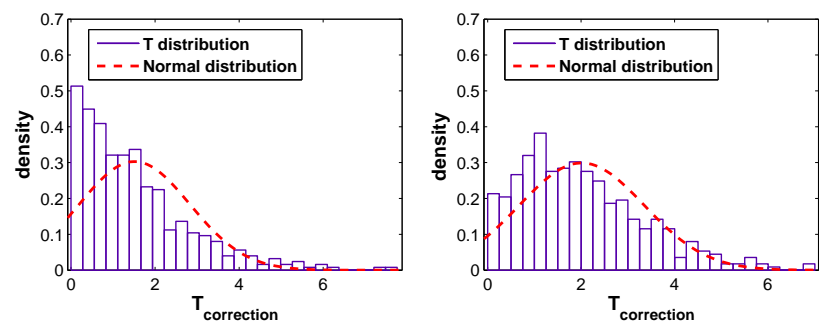

Figure 5: The distribution of $T_{\text {correction }}$ in experiment 1 (left) and experiment 2 (right). The histograms represent the distribution of $T_{\text {correction }}$, while the red curves represent the pdf (probability density function) of the normal distribution fitting onto the data.
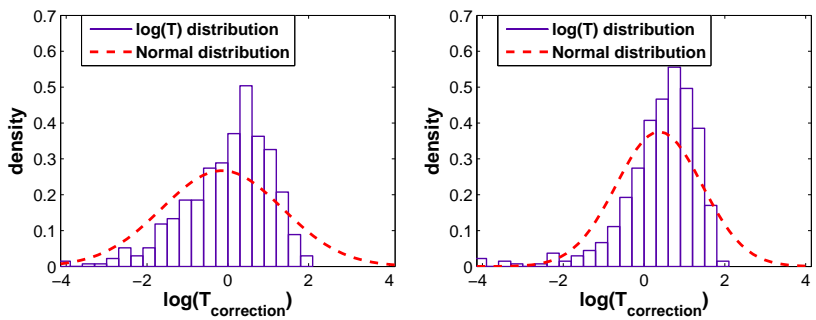

Figure 6: The distribution of $\log \left(T_{\text {correction }}\right)$ in experiment 1 (left) and experiment 2 (right). The histograms represent the distribution of $\log \left(T_{\text {correction }}\right)$, while the red curves represent the pdf of the normal distribution fitting onto the data.

\subsection{Pilot Study}

The pilot study, prior to the main experiment, was conducted with five subjects. It aims at roughly revealing how $T_{\text {correction }}$ could be modeled with $W, L$ and $v$. Intuitively, a smaller $W$, a longer $L$ or a larger $v$ is expected to result in a longer $T_{\text {correction }}$. In pilot study 1 and 2, therefore, we simply hypothesize that the relationship has the following form:

$$
\log \left(T_{\text {correction }}\right)=a+b \frac{L}{W}
$$

The idea above is based on the fact that the temporal characteristics of both pointing task (Equation 3) and steering task (Equation 6) could be successfully modeled by term $L / W$. Since the definition of $L$ and $W$ is quite similar in these interaction tasks, we assume that there should be some correlation between the correction time and $L / W$ for an object pursuit task.

Figure 7 illustrates how model 8 fits onto the data from linear paths. As shown, there is a strong linear relationship between $\log \left(T_{\text {correction }}\right)$ and $L / W$, evidenced by the fact that the linear model passes through the $95 \%$ confidence intervals of the six means and

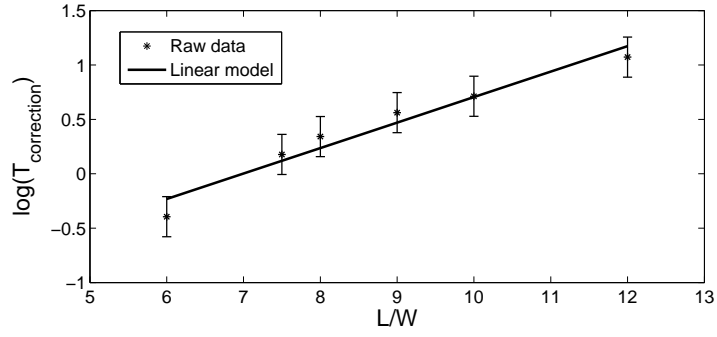

Figure 7: Pilot study 1: Linear regression between $\log \left(T_{\text {correction }}\right)$ and $L / W$ for linear paths where each asterisk represents a mean correction time of a certain $L / W$ and the corresponding error bar is its $95 \%$ confidence interval calculated using the method in [18]. The oblique line is the model fitting onto $\log \left(T_{\text {correction }}\right)$ using Equation 8.

the goodness of the fit can be evaluated by $R^{2}=0.9528$ (close to 1 ). A similar plot for pilot study 2 which studies the same $L / W$ values for circular paths is demonstrated in Figure 8. The model fits the data with $R^{2}=0.9686$.



Figure 8: Pilot study 2: Linear regression between $\log \left(T_{\text {correction }}\right)$ and $L / W$ for circular paths.

As the values selected above provide widespread representation, they were appointed as the values of $L$ and $W$ for the main experiments.

In pilot study 3 and 4 , we hypothesize a linear relationship between $\log \left(T_{\text {correction }}\right)$ and $v$ in a formula below:

$$
\log \left(T_{\text {correction }}\right)=a+b v
$$

Figure 9 and 10 specify $\log \left(T_{\text {correction }}\right)$ in terms of various values of $v$ and how Equation 9 fits onto $\log \left(T_{\text {correction }}\right)$ for linear paths and circular paths.

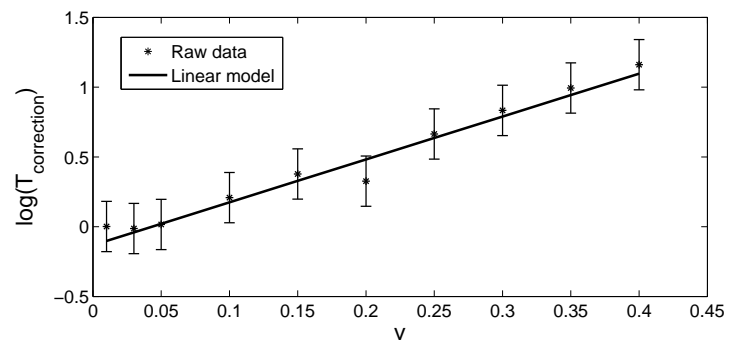

Figure 9: Pilot study 3: Linear regression between $\log \left(T_{\text {correction }}\right)$ and $v$ for linear paths. Asterisks represent the average $\log \left(T_{\text {correction }}\right)$ for different values of $v$, while the error bars represent the associated $95 \%$ confidence intervals. The oblique line is the linear model fitting onto the data using Equation 9. 


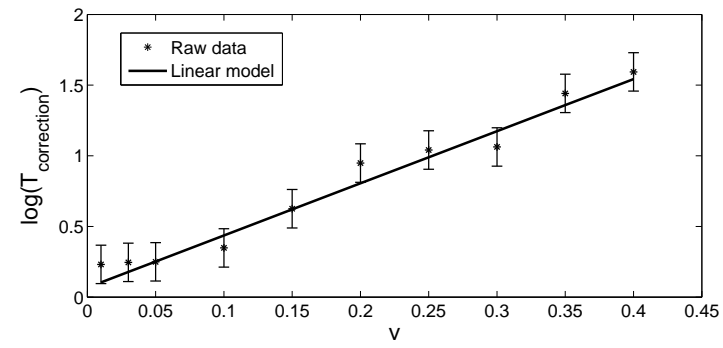

Figure 10: Pilot study 4: Linear regression between $\log \left(T_{\text {correction }}\right)$ and $v$ for circular paths.

It can be seen that there is a strong linear correlation between the $\log \left(T_{\text {correction }}\right)$ and $v$ in both linear and circular paths, evidenced by $R^{2}=0.9410$ and 0.9418 , respectively. However, as reported by the subjects, a tracking task with a target moving faster than $0.35 \mathrm{~m} / \mathrm{s}$ is too difficult to achieve, we decided to control the velocity of the moving target below $0.35 \mathrm{~m} / \mathrm{s}$. There is also a velocity threshold, as indicated in Figure 9 and 10, under which subjects find the tracking task so easy to fulfil that the correction time stays relatively constant. This threshold should fall between $0.05 \mathrm{~m} / \mathrm{s}$ and $0.1 \mathrm{~m} / \mathrm{s}$. Therefore, we chose five proper velocities for the main experiments, including $0.10,0.15,0.20,0.25,0.30 \mathrm{~m} / \mathrm{s}$.

\subsection{Main Experiment}

We have conducted two main experiments of repeated measures design, in which object pursuit tasks were examined in terms of linear (experiment 1) and circular paths (experiment 2), respectively. The main experiments, carried out with 15 subjects, were designed to evaluate the linear relationship between $\log \left(T_{\text {correction }}\right)$ and $L / W$ in Equation 8 and that between $\log \left(T_{\text {correction }}\right)$ and $v$ in Equation 9.

\subsubsection{Modeling Correction Time with $L / W$}

First of all, a repeated measures ANOVA of experiment 1 has been performed for the average correction time that arises from different values of $L / W$. The corresponding results are demonstrated in Figure 11.

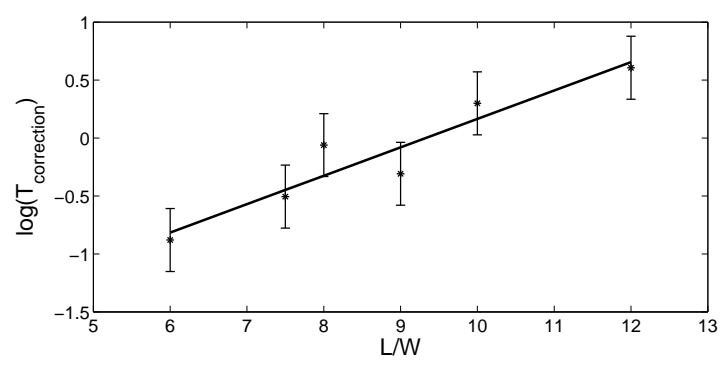

Figure 11: Experiment 1: The repeated measures ANOVA on the average correction time of different values of $L / W$ for linear paths. The error bars represent the corresponding $95 \%$ confidence interval$\mathrm{s}$. The oblique line that crosses through the confidence intervals is the linear model fitting with Equation 8.

As shown, there is a statistically significant difference between $\log \left(T_{\text {correction }}\right)$ of different values of $L / W(F(5,70)=13.922$, $p<0.0001)$. Generally speaking, the correction time has an upward tendency as the value of $L / W$ grows. This can be statistically modeled by a linear function that passes through the confidence intervals of the means as illustrated in Figure 11. A $R^{2}=0.8978$ and a $\mathrm{SSE}=0.1498$ (sum of square error) indicate a conclusive correlation between the actual data and the result of the fit. The regression parameter estimates are shown in Table 1 . The fact that the $95 \%$ confidence intervals of coefficient $b$ does not include zero illuminates that $L / W$ is an independent variable that significantly influence the correction time of the object pursuit tasks.

\begin{tabular}{|l|c|l|}
\hline Coef. & Value & {$[95 \%$ Conf. Interval] } \\
\hline$a$ & -2.287 & {$[-3.316,-1.258]$} \\
$b$ & 0.245 & {$[0.130,0.360]$} \\
\hline
\end{tabular}

Table 1: Experiment 1: regression parameter estimates on $\log \left(T_{\text {correction }}\right)$ fitting onto Equation 8 for linear paths.

A similar analysis has been done for the circular paths in experiment 2 (see Figure 12). As indicted by the results of the repeated measures ANOVA $(F(5,70)=20.225, p<0.0001)$, there is a significant difference between the $\log \left(T_{\text {correction }}\right)$ of different values of $L / W$ for circular paths as well. The change of $\log \left(T_{\text {correction }}\right)$ can be modeled by a linear function of $L / W$ shown as the oblique line in Figure 12. The goodness of the fit can be measured by $R^{2}=0.9337$ and SSE $=0.0996$. That the confidence interval of coefficient $b$ excludes zero as presented in Table 2 illustrates that $L / W$ is a statistically influential term on modeling the correction time of object pursuit tasks.

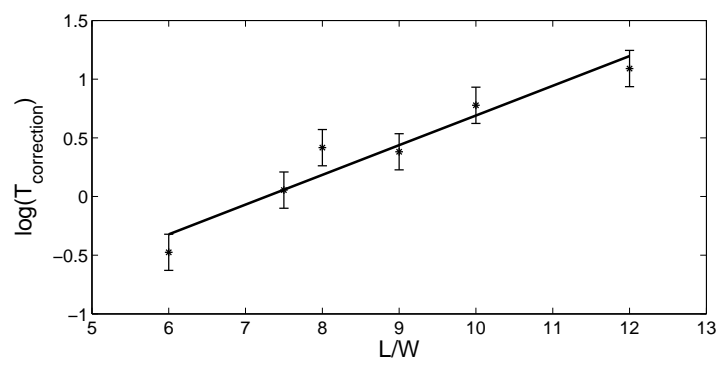

Figure 12: Experiment 2: The repeated measures ANOVA on the average correction time of different values of $L / W$ for circular paths and a linear model for correction time fitting onto Equation 8.

\begin{tabular}{|l|c|l|}
\hline Coef. & Value & {$[95 \%$ Conf. Interval] } \\
\hline$a$ & -1.841 & {$[-2.680,-1.002]$} \\
$b$ & 0.2531 & {$[0.160,0.347]$} \\
\hline
\end{tabular}

Table 2: Experiment 2: regression parameter estimates on $\log \left(T_{\text {correction }}\right)$ fitting onto Equation 8 for circular paths.

\subsubsection{Modeling Correction Time with $v$}

For the data collected from experiment 1 , we have done another repeated measures ANOVA for the average correction time of different velocities. Figure 13 shows the results. It can be seen that the difference of correction time that arises from the velocity with which the target moves is statistically significant $(F(4,56)=9.942$, $p<0.0001)$. The correction time tends to increase as the velocity rises. Consequently, it is possible to establish a linear model that passes through the confidence intervals of all the means and very much resembles the change of the correction time as velocity varies (the oblique line in Figure 13, $R^{2}=0.9255, \mathrm{SSE}=0.1178$ ). The parameters of the model in Equation 9 can be estimated using Table 3. As shown, the $95 \%$ confidence intervals of both coefficients $a$ and $b$ 


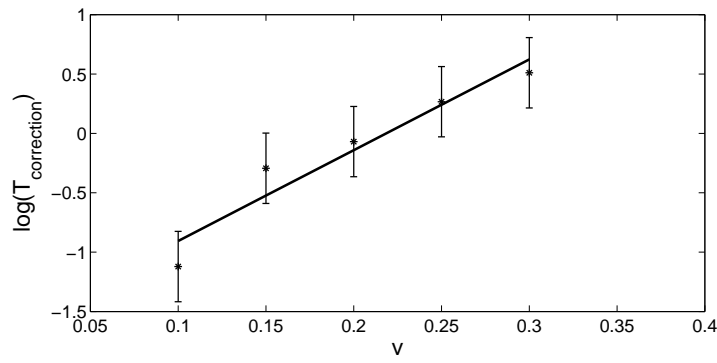

Figure 13: Experiment 1: The repeated measures ANOVA on the average correction time of different values of $v$ for linear paths. The oblique line that crosses through the confidence intervals is the linear model fitting onto Equation 9.

\begin{tabular}{|l|c|l|}
\hline Coef. & Value & {$[95 \%$ Conf. Interval] } \\
\hline$a$ & -1.671 & {$[-2.517,-0.825]$} \\
$b$ & 7.648 & {$[3.661,11.640]$} \\
\hline
\end{tabular}

Table 3: Experiment 1: regression parameter estimates on $\log \left(T_{\text {correction }}\right)$ fitting onto Equation 9 for linear paths.

exclude 0 . It indicates that velocity is definitely an influential factor in terms of correction time.

Analogous to the analysis above, the repeated measures ANOVA has been applied to the circular paths. As illustrated in Figure 14, a significant difference can be found between correction time of various velocities $(F(4,56)=15.620, p<0.0001)$. The linear model fits the data with reasonable goodness of fit $\left(R^{2}=0.8823, S S E=0.1134\right)$. The term $v$ is proved to exist on modeling the correction time of circular path object pursuit tasks, as specified in Table 4 .

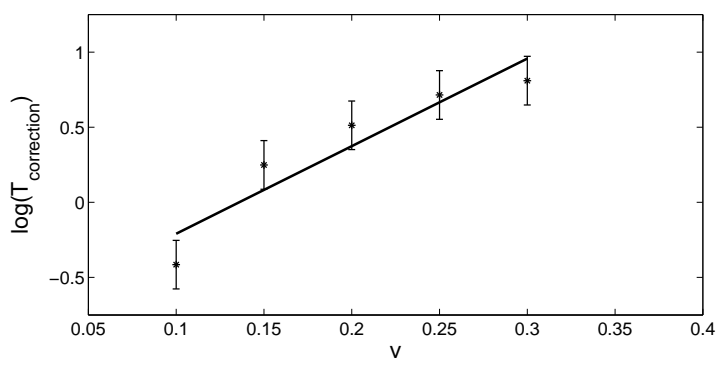

Figure 14: Experiment 2: The repeated measures ANOVA on the average correction time of different values of $v$ for circular paths. The oblique line that crosses through the confidence intervals is the linear model fitting onto Equation 9.

\subsubsection{Modeling Correction Time with $L / W$ and $v$}

As described above, there is statistically conclusive evidence showing that the correction time of the object pursuit tasks, either with a linear or circular moving target, can be successfully modeled with a linear function of $L / W$ and $v$, separately. Figure 15 shows the correction time as a function of $L / W$ for each $v$. As illustrated, the effect of $v$ is evident as a percentage increase (almost parallel) in the correction time. This indicates that there should be a possibility to construct a new model that integrates the two models (Equation 9 and 8) as shown in Equation 10:

$$
\log \left(T_{\text {correction }}\right)=a+b \frac{L}{W}+c v
$$

\begin{tabular}{|l|c|l|}
\hline Coef. & Value & {$[95 \%$ Conf. Interval $]$} \\
\hline$a$ & -0.792 & {$[-1.622,0.038]$} \\
$b$ & 5.832 & {$[1.918,9.745]$} \\
\hline
\end{tabular}

Table 4: Experiment 2: regression parameter estimates on $\log \left(T_{\text {correction }}\right)$ fitting onto Equation 9 for circular paths.

where $a, b$ and $c$ are empirically determined constants. A surface fitting, illustrated in Figure 16, is required to deal with a fitting that involves two independent variables. The surface fits the data of experiment 1 well so that the goodness of fit can be described with $R^{2}=0.9534$ and $S S E=0.8097$. Table 5 shows the corresponding parameter estimates of the surface fitting. It can be seen that no confidence interval of the coefficients includes 0 , specifying $L / W$ and $v$ are all important for modeling the correction time.

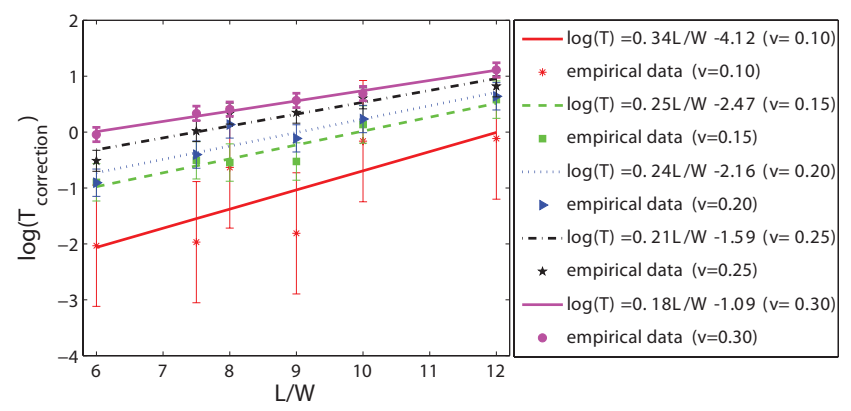

Figure 15: Experiment 1: Relationship between $\log \left(T_{\text {correction }}\right)$ and $L / W$. Points with different shapes and colors represent the raw data with different velocities, while lines represent the models $\left(\log \left(T_{\text {correction }}\right)\right.$ vs. $\left.L / W\right)$ fitting onto data with different velocities.

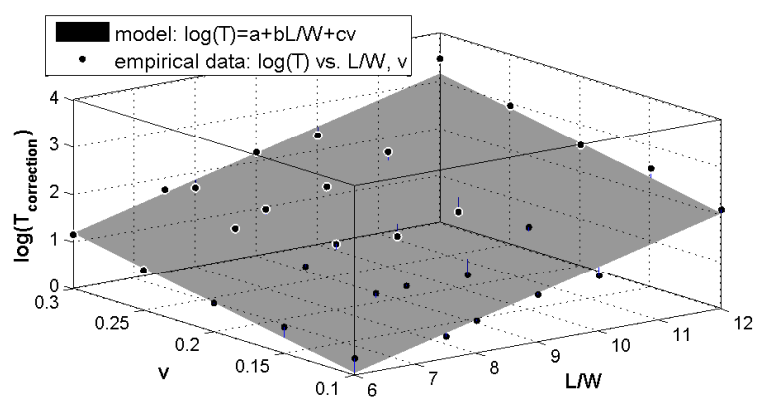

Figure 16: Equation 10 fits onto data of Experiment 1. The vertical bars represent the distances between the empirical data and the plane.

Similarly, Figure 17 demonstrates how Equation 10 fits onto the data of circular paths $\left(R^{2}=0.9553, S S E=1.1830\right)$ and Table 6 is the related parameter estimates. Both of them confirm the existence of term $L / W$ and $v$.

\section{Discussion}

Several aspects need to be discussed for the results provided above.

Fist of all, it is interesting to investigate if the total movement time $\left(T_{\text {tracking }}+T_{\text {correction }}\right)$ of an object pursuit task can be mini- 


\begin{tabular}{|l|c|l|}
\hline Coef. & Value & {$[95 \%$ Conf. Interval $]$} \\
\hline$a$ & -2.491 & {$[-2.846,-2.135]$} \\
$b$ & 0.329 & {$[0.295,0.363]$} \\
$c$ & 5.631 & {$[4.714,6.549]$} \\
\hline
\end{tabular}

Table 5: Experiment 1: the parameter estimates of surface fitting (Equation 10) onto data collected for linear paths.



Figure 17: Equation 10 fits onto data of Experiment 2.

mized. Note that the tracking time of a uniform motion pursuit task

$$
T_{\text {tracking }}=\frac{L}{v}
$$

is fixed only if $L$ and $v$ has been fixed. If we treat $v$ as a variable, $T_{\text {tracking }}$ is a monotone decreasing function of $v$. Whereas, The time for the correction phase

$$
\log \left(T_{\text {correction }}\right)=a+b \frac{L}{W}+c v
$$

is a monotone increasing function of $v$. Consequently, there should be a velocity $v$ with which $T_{\text {total }}$, of the following form

$$
T_{\text {total }}=T_{\text {tracking }}+T_{\text {correction }}=\frac{L}{v}+e^{a+b \frac{L}{W}+c v}
$$

reaches its extremum. Figure 18 demonstrates the relationship expressed by Equation 13 given $L, W, a, b$ and $c$. The optimal velocity can be derived by taking the derivative to the right side of Equation 13 in terms of $v$ and making it equal to 0 , i.e.

$$
\frac{d}{d v}\left(\frac{L}{v}+e^{a+b \frac{L}{W}+c v}\right)=0
$$

It is just to solve the function:

$$
-\frac{L}{v^{2}}+e^{a+b \frac{L}{W}} c e^{c v}=0
$$

The solution of Function 15 can be described as:

$$
v=\frac{2 \text { Product } \log \left[\frac{1}{2} c^{2} e^{-\left(a+b \frac{L}{W}\right)} \sqrt{\frac{e^{a+b} \frac{L}{W}}{c^{2} L}} L\right]}{c}
$$

\begin{tabular}{|l|c|l|}
\hline Coef. & Value & {$[95 \%$ Conf. Interval $]$} \\
\hline$a$ & -2.931 & {$[-3.361,-2.502]$} \\
$b$ & 0.407 & {$[0.366,0.448]$} \\
$c$ & 6.944 & {$[5.834,8.053]$} \\
\hline
\end{tabular}

Table 6: Experiment 2: the parameter estimates of surface fitting (Equation 10) onto data collected for circular paths.

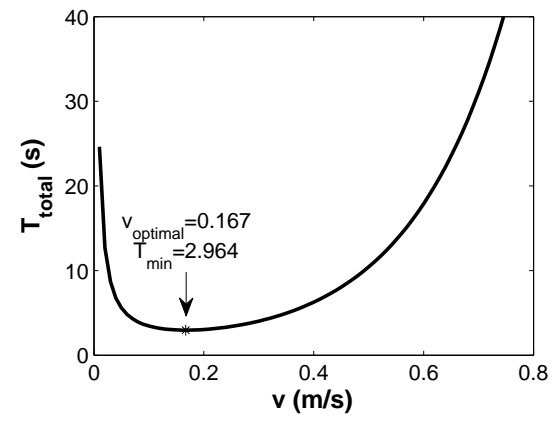

Figure 18: The relationship between the velocity $v$ and the total movement time $T_{\text {total }}$ given that $L=0.24, W=0.04, a=-2.491, b=0.329$ and $c=5.631$.

where $\operatorname{Product} \log (x)$ is the Lambert $W$ function (also called Omega function or product logarithm) which gives the principal solution for $w$ in $x=w e^{w}$ (Complex-valued solutions for $v$ have been discarded). It can be seen from Equation 16, if the target size $W$ and the path length $L$ are known, an optimal velocity to minimize the total pursuit time can be derived. This can be used as a guideline when designing user interfaces with moving targets. Assigning the specific values of $a, b$ and $c$ from Table 5 and 6 to Equation $16(L / W \in[6,12])$, we manage to approximately derive $v \in[0.093,0.167]$ and $[0.067,0.143]$ that minimizes $T_{\text {total }}$ for the object pursuit task of linear and circular paths, respectively.

Secondly, ID (index of difficulty) for a uniform motion object pursuit task needs to be defined, as in Fitts' law and steering law. We propose to define $I D$ in such a way:

$$
\log \left(T_{\text {correction }}\right)=a+b \mathrm{ID}=a+b\left(\frac{L}{W}+c^{\prime} v\right)
$$

where $c^{\prime}$ is experimentally determined constants and calculated as $c / b$ from the previous model (Equation 10). It can be seen that the definition of $I D$ above involves the effect of both $L / W$ and $v$, and $c$ ' determine the way how they influence the correction time. As calculated from Table 5 and 6, c' has approximately a value of 17 , indicating that velocity has a much bigger effect on the correction time than $L / W$ and velocity is therefore a dominant factor influencing an object pursuit task. It is very interesting to find out that a time-related factor $v$ appears in modeling an interactive task which has a non-stationary target. This breaks the rule in previous models (Fitts' law and steering law) where human temporal performance can be modeled only with spatial characteristics of the tasks.

Moreover, it is important to note that although Fitts' law, path steering law and the model we proposed (object pursuit law) describe three types of totally different interaction tasks, they have something in common. All of them present a quantitative relationship between human temporal performance and the characteristics of the tasks (both time and space-related). As experimentally verified, the relationship provides a good way to model the interaction tasks, especially the introduction of term $L / W$, which seems to be one of the most important indices for the difficulty of a task. It therefore deserves a careful examination while modeling new interaction tasks. Inspired by the fact that Fitts' law and steering law are applicable to both 2D and 3D tasks, object pursuit law, originally proposed for 3D tasks, may be also applied to the similar 2D tasks. It is sound and reasonable to predict the movement time of a $2 \mathrm{D}$ object pursuit task with the size and velocity of the moving target and the path length crossed by the target.

The last issue needs to be discussed is the restriction of the model proposed. As the tracking time always equals to the actual time that the target moves, $T_{\text {tracking }}$ is constant under such circumstances. It 
is therefore not necessary to stress the tracking time of an object pursuit task, but the correction time. Depending on the velocity with which the target moves (a uniform or variable motion), $T_{\text {correction }}$ may be influenced in different manners. The reason is that the correction time may strongly reply on the extent to which the velocity and the trajectory of the moving target is predictable. In a variable motion, however, such prediction is highly restricted. We may need the change of the velocity, i.e. the acceleration, to fully address the problem. Accordingly, to model the tracking task of a variable motion is beyond the scope of the model.

In addition, there are some factors, e.g. the curvature and orientation of the target trajectories, that are not taken into account in the model, since the number of trials required to observe the independent variables exponentially increases as the number of the variables goes up. Conducting an experiment with a large amount of trials causes much fatigue for subject, which can ultimately undermine the results of the experiment. Examining the two models for the linear and the circular paths (see Figure 16 and 17), the correction time on the circular path (curvature $\rho=1 / R$ ) is on average greater than its counterpart on the linear path $(\rho=0)$. This indicates that path curvature may play a part in the correction time and that there tends to be an increment in the correction time as the path curvature increases. Therefore, the effect of path curvature on object pursuit tasks needs to be experimentally studied in the future work.

\section{Conclusion}

In this paper, we introduced the object pursuit as an interaction task that requires subjects to continuously track a 3D target that moves with a uniform velocity in a virtual environment. A complete pursuit movement was broken into a tracking phase and a correction phase. For each phase, we proposed a model that has been experimentally verified for target moving on linear paths and circular paths. As the experimental results indicate, the tracking time is only governed by the path length crossed and the velocity with which the target moves in a uniform motion. Therefore, the tracking time is fixed once a task has been established. The correction time, however, can be modeled as a function of path length, target width and the velocity with which the target moves. As shown in Section 5, target velocity has a much bigger effect on the correction time than the target width and path length. An optimal target velocity, dependent on target width and path length, can be derived to minimize the total movement time of an object pursuit task. These could be used to design more efficient user interfaces and interaction techniques.

This is the fist attempt to model the object pursuit task in virtual environment, which is therefore of great importance. It is interesting to find out that the temporal characteristics of such a primitive interaction task can be partially described as a function of the spatial properties of the tasks, especially the existence of term $L / W$ that can also be found in Fitts' law and steering law. In addition, we proved that the time-related factor is necessary to model a task with a non-stationary target. These points could serve as guidelines for modeling a general interaction task.

\section{REFERENCES}

[1] J. Accot and S. Zhai. Beyond Fitts' law: Models for trajectory-based HCI tasks. In CHI '97: Proceedings of the SIGCHI Conference on Human Factors in Computing Systems, pages 295-302, 1997.

[2] J. Accot and S. Zhai. Performance evaluation of input devices in trajectory-based tasks: An application of the steering law. In Proceedings of the SIGCHI Conference on Human Factors in Computing Systems: the CHI is the limit, pages 466-472, 1999.

[3] A. T. Bahill and J. D. McDonald. Smooth pursuit eye movements in response to predictable target motions. Vision Research, 23(12):15731583, Apr. 1983.
[4] N. J. Cowan and E. S. Fortune. The critical role of locomotion mechanics in decoding sensory systems. The Journal of Neuroscience, 27(5):1123-1128, Jan. 2007.

[5] E. R. Crossman and P. J. Goodeve. Feedback control of handmovement and Fitts' law. The Quarterly Journal of Experimental Psychology Section A, 35(2):251-278, May 1983.

[6] C. G. Drury. Application of Fitts' law to foot-pedal design. Human Factors: The Journal of the Human Factors and Ergonomics Society, 17(4):368-373, Aug. 1975.

[7] P. M. Fitts. The information capacity of the human motor system in controlling the amplitude of movement. Journal of Experimental Psychology, 47(6):381-391, June 1954.

[8] K. Flowers. Some frequency response characteristics of parkinsonism on pursuit tracking. Brain, 101(1):19-34, 1978.

[9] D. C. Gooding, M. D. Miller, and T. R. Kwapil. Smooth pursuit eye tracking and visual fixation in psychosis-prone individuals. Psychiatry Research, 93(1):43-54, Feb. 2000.

[10] T. Grossman and R. Balakrishnan. Pointing at trivariate targets in 3D environments. In Proceedings of the SIGCHI Conference on Human Factors in Computing Systems 2004, pages 447-454, 2004.

[11] R. A. Hess. Pursuit tracking and higher levels of skill development in the human pilot. IEEE Transactions on Systems, Man, and Cybernetics, SMC-11:262-273, 1981.

[12] W. G. Iacono, M. Moreau, M. Beiser, J. A. Fleming, and T.-Y. Lin. Smooth-pursuit eye tracking in first-episode psychotic patients and their relatives. Progress in Neuro-Psychopharmacology and Biological Psychiatry, 101(1):104-116, Feb. 1992.

[13] S. G. Lisberger, E. J. Morris, and L. Tychsen. Visual motion processing and sensory-motor integration for smooth pursuit eye movements. Neuroscience, 10:97-129, Mar. 1987.

[14] L. Liu, J. B. O. S. Martens, and R. van Liere. Revisiting path steering for 3D manipulation tasks. In Proceedings of the 2010 IEEE Symposium on 3D User Interfaces (3DUI), pages 39-46, Mar. 2010.

[15] L. Liu and R. van Liere. Designing 3D selection techniques using ballistic and corrective movements. In Proceedings of Eurographics Symposium on Virtual Environments 2009, pages 1-9, 2009.

[16] I. S. MacKenzie. Fitts' law as a research and design tool in humancomputer interaction. Human-Computer Interaction, 7(1):91-139, Mar. 1992

[17] I. S. MacKenzie, A. Sellen, and W. A. S. Buxton. A comparison of input devices in element pointing and dragging tasks. In Proceedings of the SIGCHI Conference on Human Factors in Computing Systems: Reaching through technology, pages 161-166, 1991.

[18] M. E. Masson and G. R. Loftus. Using confidence intervals for graphically based data interpretation. Canadian Journal of Experimental Psychology, 57:203-220, 2003.

[19] G. K. J. Miller and D. R. Riley. The effect of visual-motion time delays on pilot performance in a pursuit tracking task. In Visual and Motion Simulation Conference, Jan. 1976.

[20] J. Raskin. The humane interface: New directions for designing interactive systems. ACM Press/Addison-Wesley Publishing Co., 2000.

[21] P. Soliveri, R. G. Brown, M. Jahanshahi, T. Caraceni, and C. D. Marsden. Learning manual pursuit tracking skills in patients with parkinson's disease. Brain, 120(8):1325-1337, 1997.

[22] R. W. Soukoreff and I. S. MacKenzie. Towards a standard for pointing device evaluation, perspectives on 27 years of Fitts' law research in HCI. International Journal of Human-Computer Studies, 61(6):751789, Dec. 2004.

[23] A. Steed. A simple method for estimating the latency of interactive, real-time graphics simulations. In Proceedings of the 2008 ACM symposium on Virtual reality software and technology, pages 123-129, 2008.

[24] R. J. Teather, A. Pavlovych, W. Stuerzlinger, and I. S. MacKenzie. Effects of tracking technology, latency, and spatial jitter on object movement. In Proceedings of IEEE Symposium on $3 D$ User Interfaces 2009, pages 43-50, 2009.

[25] S. Zhai, J. Accot, and R. Woltjer. Human action laws in electronic virtual worlds: An empirical study of path steering performance in VR. Presence: Teleoperators and Virtual Environments, 13(2):113 127, 2004. 